\title{
A Visualization Solution of the Stress State of Connecting-rod Big End in the Experimental Device for Lubricating Condition of the Connecting-rod Big End Bearing
}

\author{
Tran Thi Thanh Hai*, Le Anh Dung, Do Tien Dat \\ Hanoi University of Science and Technology, No. 1, Dai Co Viet, Hai Ba Trung, Hanoi, Viet Nam \\ Received: August 19, 2020; Accepted: November 12, 2020
}

\begin{abstract}
This paper presents a visualization solution of the stress state of the connecting-rod big end in the experimental device for lubricating of the connecting-rod big end bearing. The connecting-rod model is in photoelastic material that is subjected to the load simulation corresponding to the engine's operating cycle. This experimental device and the connecting-rod model are used to determine the load diagram, measure the oil film pressure, oil film thickness, oil film temperature. In this study, a visualization system of stress state in the connecting-rod big end is built that stress of connecting-rod is one of the characteristics when we study the connecting-rod big end bearing. The stress state of the connecting-rod is visualized by the transmission photoelasticimetry. This method allows the visualization of the isochrones fringes, which are lines of equal difference regarding the main stress in the connecting-rod. The visualization system consists of a polarizer, two quarter ware blades, and an analyzer. The stress contour's images of the connecting-rod at different angles of the crankshaft are realized by a CCD camera. The stress state in the connecting-rod is compatible with the load applied.
\end{abstract}

Keywords: Connecting-rod, bearing, stress, photoelasticimetry, isochrones fringe

\section{Introduction}

The lubrication of connecting-rod big end bearing is an important problem for the operation of engines. Several parameters have large influences on the behavior of the oil film and the solids [1-6]. A special device was built to study the lubrication of the connecting-rod bearing. The load applied to the connecting-rod and the pressure in the lubricant oil film change the stress in the connecting-rod during the operating cycle. This problem is one of the characteristics we need to consider when studying the connecting-rod big end bearing. In this study, we propose a visualization solution of the stress state in the experimental device.

The connecting-rod big end model in the experimental device is made of photoelastic material that is subjected to the load simulation corresponding to the engine's operating cycle. A method to visualize the stress state of connecting rod big end is based on some classical principles of physics. The photoelasticimetry is an experimental method allowing to visualize the constraints existing inside a solid thanks to its photoelasticity. It is a mainly optic method based on the birefringence on materials subjected to stresses.

* Corresponding author: Tel.: (+84) 978263926

Email: hai.tranthithanh@hust.edu.vn

\section{Theory of photoelasticity}

Many transparent noncrystalline materials that are optically isotropic when free of stress become optically anisotropic and display characteristics similar to crystals when they are stressed. This behavior is known as temporary double refraction, an effect that an isotropic material can become birefringent (anisotropic), when placed under stress. The optical axis is in the direction of the stress and induced birefringence is proportional to the stress. The method utilizes a birefringent model of the actual structure to view the stress contours due to external loading or residual birefringence. When white light is used for illumination, a colorful fringe pattern reveals the stress/strain distribution in the part. By utilizing a monochromatic light source as illumination, it enables a better definition of fringes especially in areas with dense fringes as at stress concentration points [7-8].

\subsection{Different process of photoelasticimetry}

To measure the stresses, several methods are used:

1. A planar reproduction of the studied shape can be made, cut out of a photoelastic material. This model is observed by transparency and placed between two polarizing filters (Fig.1), while forces are applied to it. 


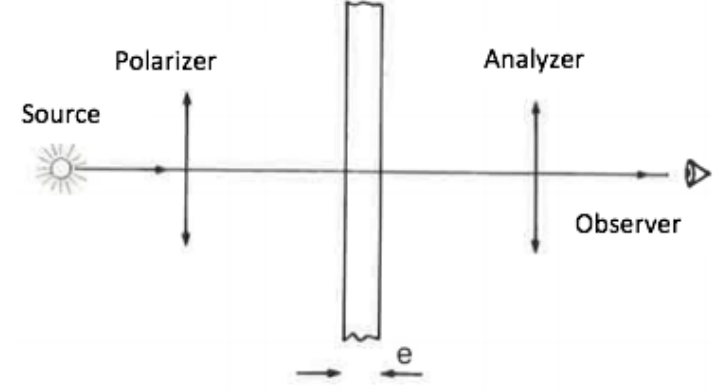

Fig. 1. Transmission photoelasticimetry

2. It is also possible to cover a real structure with a thin layer of photoelastic product. The structure is not transparent, in general, the surface is made reflective using a paint or an adhesive loaded with metal powder. Light passes through the photoelastic coating, is reflected and passes through the coating a second time (Fig.2). The polarizing filters are placed side by side.

The theories of photoelasticity are identical, whether they are instruments by transparency or by reflection. Simply, it should be noted that, by reflection, the thickness of the coating counts 2 times. The thickness of a model observed by transparency is $e$, the double of the thickness of a coating observed by reflection is $2 t$.

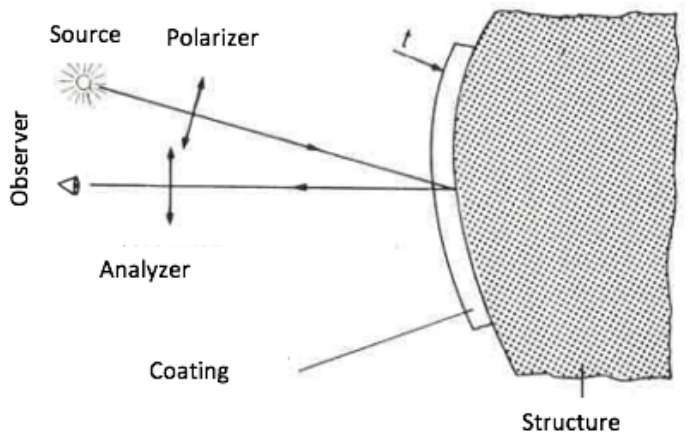

Fig. 2. Photoelasticity by reflection

\subsection{Fundamental laws of photoelasticity}

With the case in Fig.1, the force is applied to the model, which creates stress. The relationships between stresses and accidental birefringence are linear. The relation of stresses is the law of Maxwell and the law of Neumann is used for strains. The optical delay of one of the light components respect to the other is proportional to the material crossed thickness to the difference of the principal stresses or to the difference of the principal deformations.

$$
\begin{aligned}
& \text { Maxwell } \delta=\text { C.e. }\left(\sigma_{1}-\sigma_{2}\right) \\
& \text { Neumann } \delta=\text { K.e. }\left(\varepsilon_{1}-\varepsilon_{2}\right)
\end{aligned}
$$

Where $\sigma_{1}, \sigma_{2}$ and $\varepsilon_{1}, \varepsilon_{2}$ are the principal stresses and the deformation at a point; $C$ is the stress optic coefficient and is measured in Brewsters. This unit corresponds to a delay of 1 Angstroem caused by stress of 1 bar when the light passes through $1 \mathrm{~mm}$ thick of the photoelastic material. In CGS units or in system SI:

$$
\text { 1Brewster }=10^{-13} \frac{\mathrm{cm}^{2}}{\text { dyne }}=10^{-12} \frac{\mathrm{m}^{2}}{\text { Newton }}
$$

In the elastic domain, $E_{P}$ and $\mu_{P}$ are the Young's modulus and the Poisson's ratio of the photoelastic material.

$$
K=\frac{C E_{p}}{1+\mu_{p}}
$$

The length $\delta$ is the delay of one light component on the other, along their common path. Expressed as a phase angle $\varphi$, it is valid, for a wavelength $\lambda$ of light:

$$
\varphi=2 \pi \frac{\delta}{\lambda}
$$

\subsection{Effects of birefringence on light}

A light of plane polarization (Fig.3)[8] appears in the form:

$$
x=a \cos w t
$$

This light passes through a photoelastic medium, its plane of polarization being inclined at an angle $\beta$ on one of the main directions, then the analyzer polaroid.

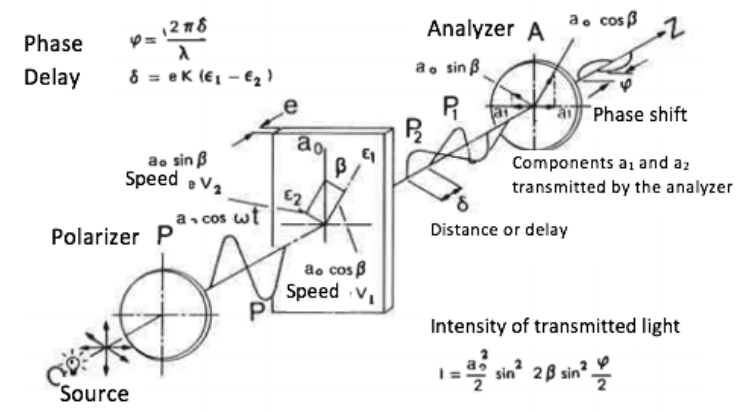

Fig.3. Flat light passing through a constrained plastic [8].

The remaining light intensity varies as:

$$
\sin ^{2} 2 \beta \sin ^{2} \frac{\varphi}{2}=\sin ^{2} 2 \beta \sin ^{2} \pi \frac{e K}{\lambda}\left(\varepsilon_{1}-\varepsilon_{2}\right)
$$

This light intensity can be zero for two reasons:

1. When $\beta=0$ or $\beta=\pi / 2$, i.e. when the polarizers are parallel to the principal directions of the stresses. This property is independent of the wavelength, thickness and sensitivity of the plastic. It is used to demarcate the main directions at each point of a model. When the points which have principal stress directions parallel and perpendicular to a given 
direction thus appear in the form of a black line called isocline.

$$
\text { 2. When }\left(\varepsilon_{1}-\varepsilon_{2}\right)=n . f
$$

with $f=\frac{\lambda}{e . K}$ and $n$ being any integer, that means when the difference of the main strains is zero or equal to an integer multiple of a fixed value $f$. If therefore we consider a point subjected to increasing deformations, it will be alternately black and luminous, the variation in luminous intensity being a sinusoidal function of the deformation. Conversely if, at a fixed load, one observes the whole of a structure, all the points for which the difference of the principal deformations is zero or equal to an integer multiple of a fixed value are black. Black fringes appear, locus of the points for which, $\left(\varepsilon_{1}-\varepsilon_{2}\right)=n$.f, $n$ is an integer. This is monochromatic light.

The isoclines were, therefore, very useful in locating the main directions. They interfere with the observation of the other fringes, the isochrones which will be used to measure the stresses. The isoclines could be eliminated by rotating the polaroids very quickly using a mechanical device. By retinal persistence, we would see only isochrones.

There is a more elegant solution for rotating the plane of polarization at high speed. For this, it suffices to place, on the path of the incident light, a birefringent body oriented at $45^{\circ}$ with respect to the direction of polarization, and creating an optical delay equal to a quarter of a wavelength (Fig.4) [8]. This amount, in the calculation which gave the relation (7) to be replaced in one of the components, a sine or a cosine. The light is then circular. However, as the presence of the quarter wave has modified the total birefringence, this effect is compensated by placing, before the analyzer, a second quarter wave oriented perpendicular to the first, which cancels out the previous disturbances.

\section{Visualization system the stress of connecting- rod big end in the experimental device}

The experimental device (Fig.5a) respects the kinematics of connecting-rod crank system. The connecting-rod model is formed by a rigid small end (8) and a big end in photoelastic material (9a and 9b) [9] [10]. The connecting-rod is subjected to the simulation load as in the engine and is immersed in an oil chamber.

The studied connecting-rod big end formed by a body (9a), a cap (9b) and the journal (10), all forms a smooth bearing. The oil feeding system for studying bearing consists of an oil tank (17), a hydraulic pump (18), a rotating distributor and one distribution channel which crosses all along the length of the crankshaft. This experimental device and the connecting-rod model are used to determine the load diagram, measure the oil film pressure, oil film thickness, oil film temperature. In this study, we built a system to visualize the state of stress of connectingrod big end. Fig.5b presents the connecting-rod model in the experimental device.

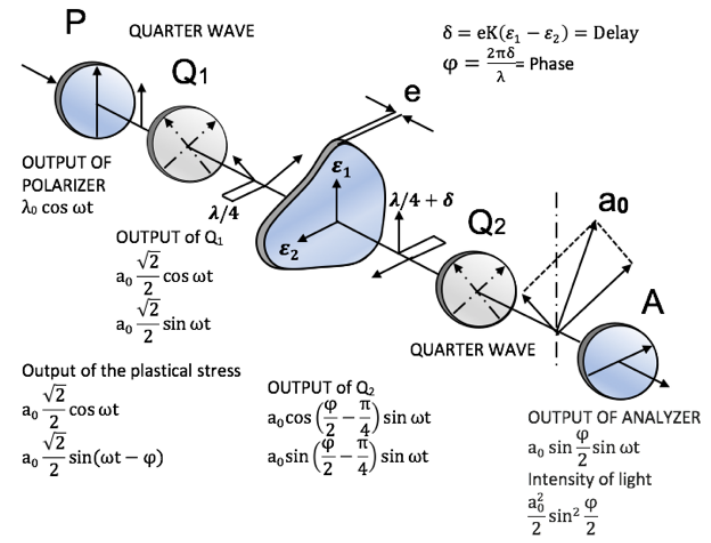

Fig. 4. Circular light [8]

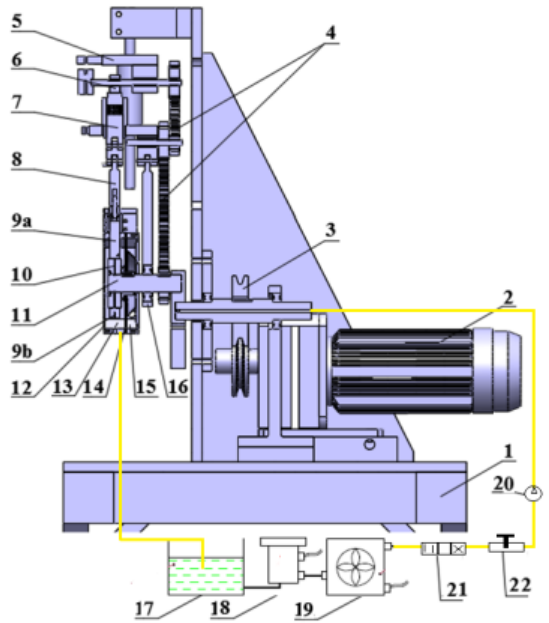

a)

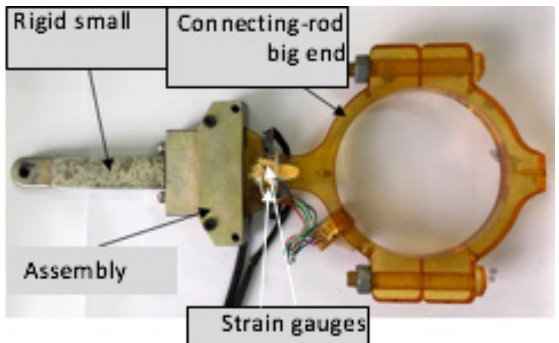

b)

Fig. 5. a. Funtional scheme of experimental device; b. The connecting-rod model

With the theory of photoelasticity was presented in part 2, we use one polariscope (or analyzer) and one $1 / 4$ wave blade are integrated into one plate, use sheets where one side was the polariscope (or analyzer) and the other side was the $1 / 4$ wave blade. Fig. 6 presents the optical assembly scheme used for the visualization isochrones fields in the connectingrod big end in the experimental device. 
For visualizing the isochrones field of the connecting-rod big end bearing, a lighting system is built. The light source consists of four halogen lamps that are located in a chamber. This light system is placed in a room behind the oil chamber (Fig.7). A CCD camera - Basler acA1300-200um support follows the same movement that the connecting-rod and thus makes it possible to photograph in detail the connecting-rod during the functional cycle (Fig.8).

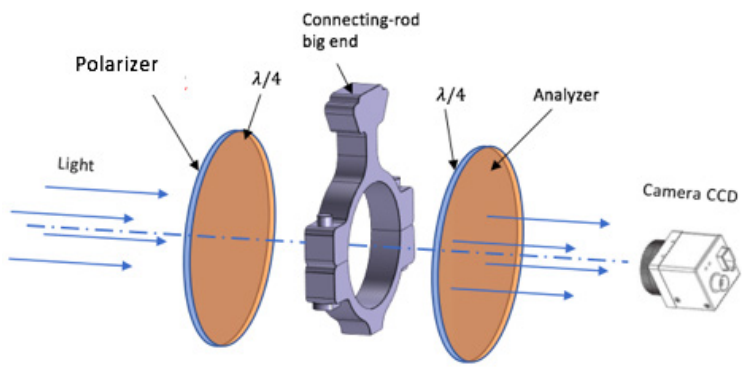

Fig. 6. Visualization system for measuring the stress of connecting-rod big

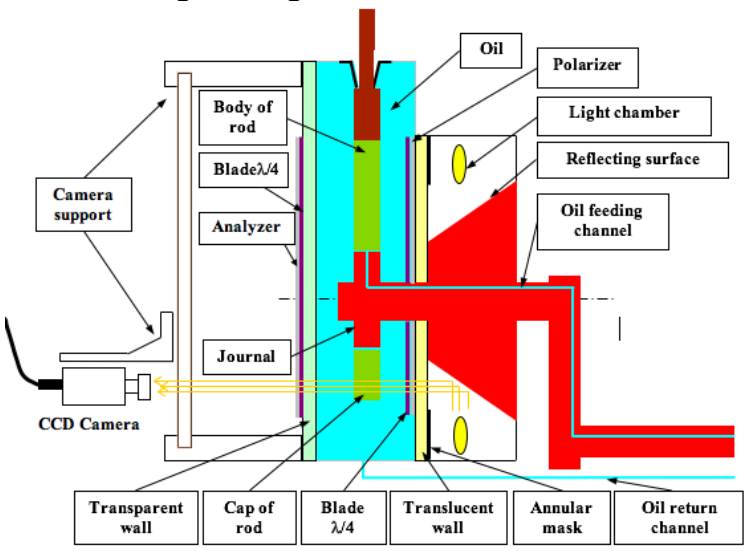

Fig. 7. The lighting system, oil feeding channel

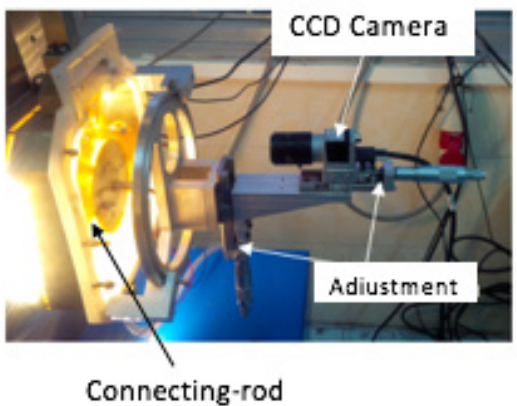

Fig. 8. CCD camera system

Table 1 and Table 2 present the connecting-rod big end bearing parameters and characteristics of the big-end material. The lubricating oil of the bearing is silicone oil Belsil F100. This oil is transparent so it does not affect image quality. The lubricating oil characteristics are shown in Table 3.
Table 1. Connecting-rod big end bearing parameters

\begin{tabular}{|l|l|}
\hline Rotational Frequency (rpm) & 0 to 250 \\
\hline Bearing diameter (mm) & 97 \\
\hline Bearing radial clearance (mm) & 0,3 \\
\hline Connecting-rod thickness (mm) & 20 \\
\hline Length of the connecting-rod (mm) & 257 \\
\hline
\end{tabular}

Table 2. Characteristics of the connecting-rod

\begin{tabular}{|l|c|}
\hline Density $\rho_{c}\left(\mathrm{Kg} / \mathrm{m}^{3}\right)$ & 1200 \\
\hline Young modulus $E_{c}(\mathrm{MPa})$ & 3150 \\
\hline $\begin{array}{l}\text { Coefficient of thermal expansion } \alpha_{c} \\
(1 / \mathrm{K})\end{array}$ & $22.10^{-6}$ \\
\hline Thermal conductivity $k_{c}(\mathrm{~W} / \mathrm{m} . \mathrm{K})$ & 0.18828 \\
\hline Poisson coefficient & 0.36 \\
\hline $\begin{array}{l}\text { Photoelastic constant of PLM-4R } v_{c} \\
(\mathrm{kPa} / \text { fringe/m) }\end{array}$ & 0.32 \\
\hline
\end{tabular}

Table 3. Characteristics of Belsil F100 silicone oil

\begin{tabular}{|l|c|}
\hline Density $\rho_{s}\left(\mathrm{Kg} / \mathrm{m}^{3}\right)$ & 980 \\
\hline Viscosity $\mu_{0}$ at $40^{\circ} \mathrm{C}(\mathrm{Pa} . \mathrm{s})$ & 0.33 \\
\hline
\end{tabular}

Place two sheets before and after the connecting-rod as shown in Fig.6 and turn on the light source, we obtain the colourful fringe pattern reveals the stress/strain distribution of the connectingrod big end in Fig.9. The CCD camera can be rotated 360 degrees to take images at the different boring areas. That means the isochrones field on the whole of the connecting-rod is obtained by the embarked CCD camera which allows precise positioning of the camera. The CCD camera using the image acquisition and processing software can photograph images. Fig. 10 presents the isochrones field at the $330^{\circ}$ area of boring, one of the images is the original image (a) and the other image (b) at the $360^{\circ}$ crank angle for a rotation speed of $100 \mathrm{rpm}$. It clearly shows the influence of dynamic loading.

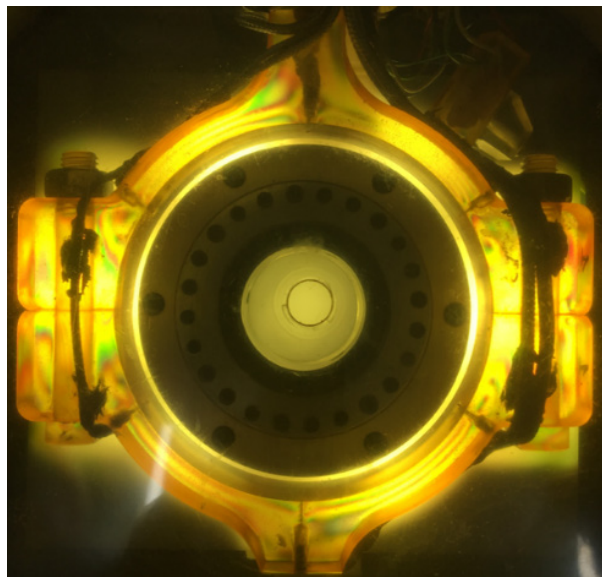

Fig. 9. Colourful fringe of the connecting-rod big end bearing 


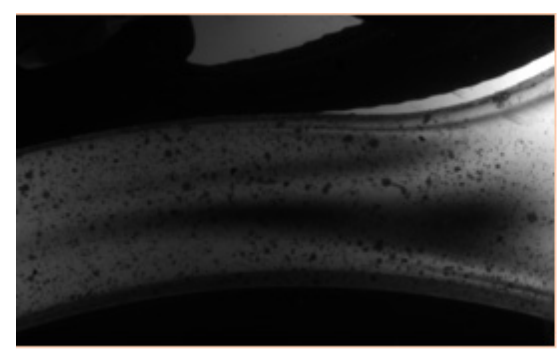

a) Reference

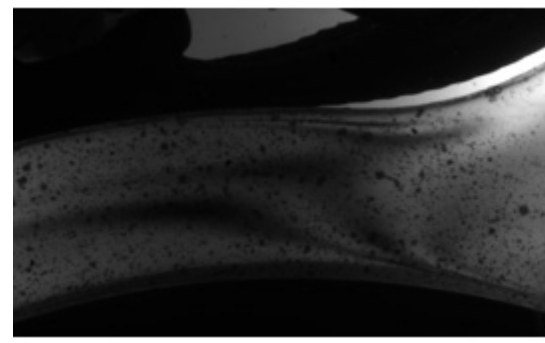

b) Dynamic

image

Fig. 10. Isochrones field at $330^{\circ}$ of housing for the crank angle $360^{\circ}, 100 \mathrm{rpm}$

\section{The experimental visualization state stress of connecting-rod}

When the experimental device is working, under the load applied to the connecting-rod and the pressure in the lubricant oil film, the stress changes in the connecting-rod during the operating cycle. Fig.11 presents the load diagrams at a rotation speed of 80 rpm and $100 \mathrm{rpm}, 150 \mathrm{rpm}$, and $180 \mathrm{rpm}$.

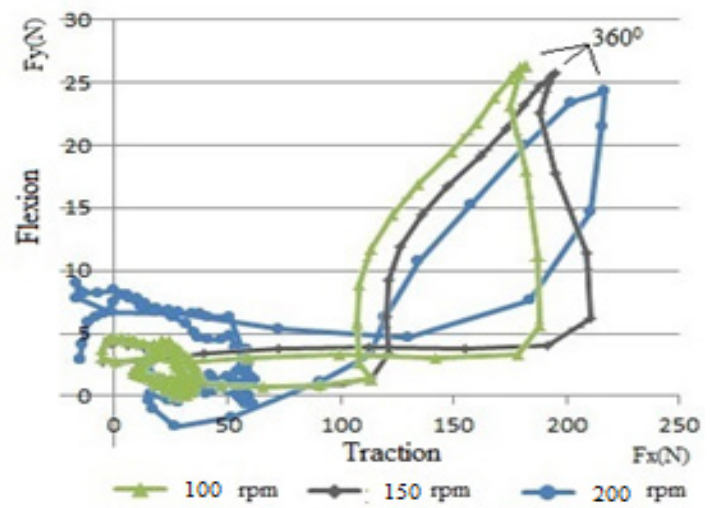

Fig. 11. Load diagrams at the rotation speed of 100 rpm and $150 \mathrm{rpm}$ and $180 \mathrm{rpm}$

The CCD camera can be rotated 360 degrees to take images at the different boring areas. That means the isochrones field on the whole of the connectingrod is obtained by the embarked CCD camera which allows precise positioning of the camera. The CCD camera using the image acquisition and processing software can photograph images. The images of the different areas are repositioned using Adobe Photoshop CS6 software to obtain the total isochrones field of the big-end bearing.
Fig.12 represents the fields of connecting-rods in the stationary state and at the $360^{\circ}$ of the crankshaft (time of the explosion). The isochrones fields are obtained from the images taken with steps of $30^{\circ}$ angle of the boring for the same crank angle $360^{\circ}$. It shows that, depending on the position of the housing bearing, the contours are darker or change the fringes. At the $0^{\circ}$ area of housing bearing, the contours are most variable. It is reasonable because this area is the maximum charge zone. In the opposite area $\left(180^{\circ}\right.$ of boring), the fringes vary little, this can be explained due to the lowest load.

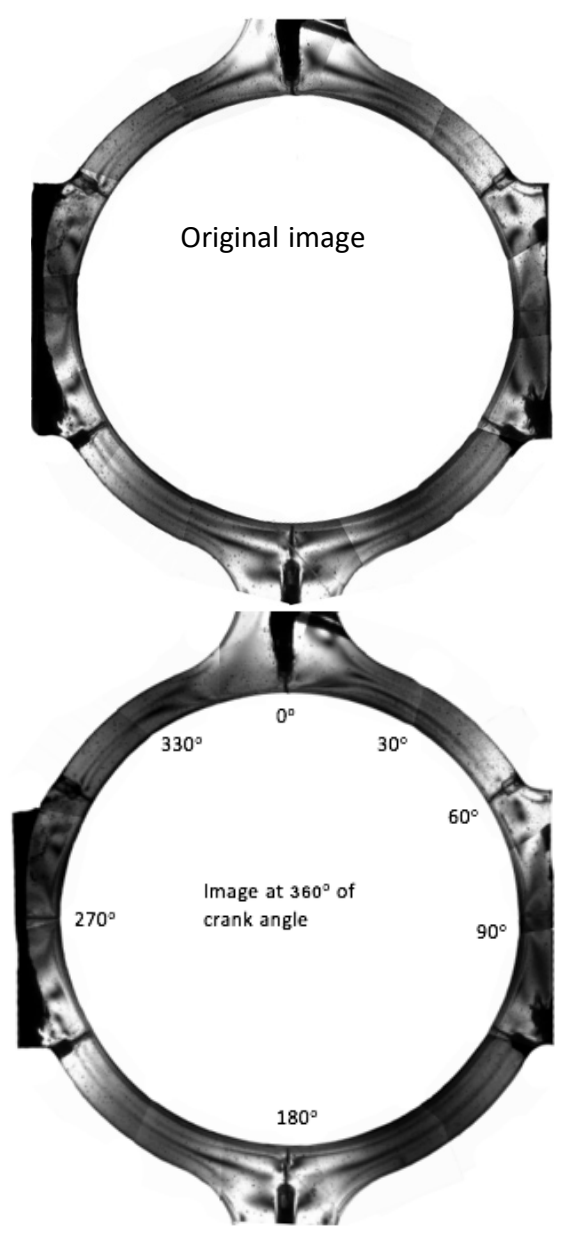

Fig. 12. Bearing isochrones field in the stationary state and at the $360^{\circ}$ of crank angle, $100 \mathrm{rpm}$

\section{Conclusion}

The stress of the connection-rod is one of the characteristics when we study the connecting-rod big end bearing. For this reason, we have built a visualization system to visualize the stress state of the connecting-rod big end in the experimental device for lubricating of the connecting-rod big end bearing.

The stress state of connecting-rod in the experimental device is visualized by the transmission photoelasticimetry method. The method utilizes a 
birefringent model of the actual structure to view the stress contours due to external loading or residual birefringence. Using monochromatic light enables a better definition of fringes especially in areas with dense fringes as at stress concentration points. The visualization system consists of a polarizer, twoquarter blades, and an analyzer.

During the operating of the experimental device, under the load applied to the connecting-rod and the pressure in the lubricant oil film, the stress in the connecting-rod is changed. The isochrones field on the whole of the connecting-rod is obtained by the embarked CCD camera which allows precise positioning of the camera. The CCD camera using the image acquisition and processing software can photograph images. The stress state in the connecting-rod is compatible with the applied load. At the $360^{\circ}$ angle of the crankshaft, a sudden change of the fringes on the rod reflects the maximum load applied at the time of the explosion. At the other angles of the crankshaft, the fringes vary little, this can be explained due to the lower load.

\section{Acknowledgements.}

This research is funded by the Hanoi University of Science and Technology (HUST) under project number T2018-PC-025

\section{References}

[1] Moreau H, 2001, Mesures des Epaisseurs du Film d'Huile dans les Paliers de Moteur Automobile et Comparaisons avec les Résultats Théoriques, Thèse de Doctorat de Université de Poitiers

[2] F.M. Meng, Y.Y. Zhang, Y.Z. Hu, 2007, The effect of compliance on peak oil film pressure in connecting- rod bearing, Tribology international 40 , 1089-1099.

[3] H. Shahmohamadi., 2014, Big End Bearing Losses with Thermal Cavitation Flow Under Cylinder Deactivation, Tribol Lett (2015).

[4] H. Chamani., 2015, Thermo-elasto-hydrodynamic (TEHD) analysis of oil film lubrication in big end bearing of a diesel engine. Vol. 5, No. 1, Aut. Win. 2015-16.

[5] Norbert Lorenz., 2017, Thermal analysis of hydrodynamic lubricated journal bearings in internal combustion engines. Proc IMechE Part K: J Multibody Dynamics.

[6] Tran Thi Thanh Hai, Nguyen Dinh Tan, Luu Trong Thuan, 2019, Influence of the radial clearance on the pressure distribution of the 5S-FE engine's connecting-rod big end bearing, Journal of Science and Technology Technical University, No 132 (2019), pp.40-45.

[7] Avril J., 1975, Encyclopédie Vishay d'Analyse des Contraintes, Edition Vishay, Malakoff.

[8] Wei-Chih Wang, Photoelasticity, Lecture material, University of Washington.

[9] Tran Thi Thanh Hai, 2018, A solution for measuring the oil film pressure of the connecting-rod big end bearing in the experimental device, The University of Danang Journal of Science and Technology, 11/2018 (1), 22-25.

[10] Tran Thi Thanh Hai, 2018, A Solution for Creating the Simulating Load on Connecting-Rod in the Experimental Device for Lubricating Condition of the Connecting-Rod Big End Bearing, Journal of Science and Technology Technical university, No 129, pp.1520. 